\title{
Ein Beitrag zur Kenntnis der Pentosurie vom chemischen Standpunkt \\ von
}

\author{
Ernst Zerner und Rudolfine Waltuch. \\ Aus dem II. chemischen Universitätslaboratorium in Wien.
}

(Vorgelegt in der Sitzung am 10. Juli 1913.)

Seitdem Salkowski ${ }^{1}$ im Jahre 1892 die Pentosurie entdeckte, ist diese interessante Anomalie des Stoffwechsels wiederholt Gegenstand des Studiums gewesen. Der Entdecker selbst konnte konstatieren, daß der Harn hierbei reduziere, aber weder drehe noch gärungsfähig sei und stellte die Pentosennatur des Zuckers durch Bereitung und Analyse des Osazons fest. In zwei weiteren Pentosuriefällen wurden die gleichen Erscheinungen wahrgenommen. Eine eventuell vorhandene Aktivität des Osazons konnte nicht wahrgenommen werden, da eine 0.4 prozentige alkoholische Lösung bereits für die Polarisation zu dunkel war.

Acht Jahre später hat sich $\mathrm{Neuberg}{ }^{2}$ eingehend mit dem Gegenstand beschäftigt und es ist ihm gelungen, in unanfechtbarer Weise an einem der Salkowski'schen Fälle festzustellen, daß der Harn $d$, l-Arabinose enthielt. Dieselbe konnte nicht nur durch Derivate identifiziert, sondern auch in Substanz dargestellt werden und erwies sich in jeder Beziehung mit einem von O.Ruff zur Verfügung gestellten Präparat von $d, l$-Arabinose identisch. Die Frage schien also damit gelöst zu sein und tatsächlich ist in die meisten Lehrbücher der Satz übergegangen, daß bei Pentosurie $d, l$-Arabinose im Harn ausgeschieden werde.

1 Zusammenfassende Mitteilung, H. 27, 507 (1899).

2 B. 33,2243 ff. (1900). 
Indessen zeigte sich bald, daß der Neuberg'sche Befund auf Verallgemeinerung keinen Anspruch erheben dürfe. Denn kurz nach dem Erscheinen der erwäbnten Arbeit fand R. Luzzatto, ${ }^{1}$ bei der im Hofmeister'schen Laboratorium ausgeführten Untersuchung eines Pentoseharnes, daß sich aus demselben ein rechtsdrehendes Osazon gewinnen lasse. Eine quantitative Bestimmung der Drehung ergab annähernd den Wert, welchen Neuberg im Pyridin-Alkoholgemisch für $l$-Arabinosazon gefunden hatte. Eine Isolierung des Zuckers in der von Neuberg angegebenen Weise konnte nicht erzielt werden, ebensowenig gelang es, die Drehung des Zuckers selbst mit Sicherheit zu ermitteln. Luz z atto konnte diesbezüglich bloß in einigen Fällen am konzentrierten Harn Rechtsdrehung beobachten. Er zog aus seinen experimentellen Resultaten den Schluß, daß in diesem Fall $l$-Arabinose im Hain enthalten gewesen sei.

In der Folgezeit haben sich eine Reihe von Forschern, vornehmlich aus medizinischen Kreisen und insbesondere vom klinischen Standpunkt mit der Pentosurie beschäftigt. Wir können auf eine genauere Wiedergabe der bezüglichen Literatur verzichten, da diese Untersuchungen vom chemischen Standpunkt nichts Neues bieten; auch die Anführung der Literaturstellen scheint uns überflüssig, da in der gleich ausführlich zu besprechenden Arbeit von Klercker ${ }^{2}$ eine sehr gründliche Zusammenfassung der einschlägigen Forschungen gegeben ist. Wir möchten nur hervorheben, daß in nahezu allen Fällen inaktive Harne gefunden wurden. Nur Brat und Blumental, ${ }^{3}$ Rosenfeld ${ }^{4}$ und Schüle $r^{5}$ haben Harne mit nicht unbedeutender Rechtsdrehung gefunden, die vielleicht, wie wir mit Klercker annehmen wollen, auf gleichzeitige Anwesenheit von $d$-Glukose zurückzuführen sein könnte; zumindest ist dies nicht von den Autoren in einwandfreier Weise ausgeschlossen worden. Von den beiden ersteren Forschern wurde ein rechtsdrehendes

1 Beiträge zur chem. Physiol, und Pathol., VI, 87 (1905).

2 Deutsehes Archiv für klin. Medizin, 108, $277 \mathrm{ff}$. (1912).

3 Deutsche Klinik, 1902 ; zitiert nach Klercker. Das Original konnten wir uns nicht beschaffen.

4 Medizin. Klinik, 1906, II, 1041.

5 Berl. klin. Woch., 1910, II, 1322. 
Osazon aus dem Harn dargestellt, aber leider die Größe der Drehung nicht angegeben. Andrerseits haben O. und R. Adler ${ }^{1}$ sowohl als auch Blum² je einen Pentosuriefall mit linksdrehendem Harn beschrieben, wobei die Linksdrehung vielleicht auf gepaarte Glukuronsäuren zurückzuführen ist. Im ersten Fall wurde auch das Osazon im Polarisationsapparat untersucht und gefunden, daß es in alkoholischer Lösung zunächst rechts dreht, die Drehung aber nach einiger Zeit verschwindet.

Daß in allen diesen Fällen wirklich Pentosurie vorlag, ist klinisch dadurch, daß die Zuckerausscheidung im Harn alimentär nicht zu beeinflussen war und chemisch insofern erwiesen worden, als der Harn die bekannten Pentosereaktionen lieferte und insbesondere ein Osazon vom ungefähren Schmelzpunkt $160^{\circ}$ (die Angaben differieren von 150 bis $163^{\circ}$ ) gab, das in vielen Fällen auch auf analytischem Wege als Pentosazon sichergestellt wurde. Leider ist aber, wie gesagt, in den meisten Fällen nur der Harn, nicht aber auch das Osazon polarimetrisch geprüft worden.

Nur zwei Arbeiten wollen wir ganz besonders hervorheben. Die eine ist in allerjüngster Zeit von Elliot und Raper ${ }^{3}$ ausgeführt worden. Diese Autoren haben sich gleich Luzzatto bemüht, mittels der Diphenylhydrazinmethode von Neuberg, den Zucker zu isolieren, ohne zu einem Resultat zu gelangen, wiewohl sie die von Neuberg angegebenen Versuchsbedingungen aufs genaueste eingehalten haben. Das Osazon, welches sie aus dem Harn gewannen (Schmelzpunkt $163^{\circ}$ ), gab weder mit $l$-Arabinosazon noch mit $d$-Ribosazon (Präparat von Levene und Jakobs) genischt eine wahrnehmbare Schmelzpunktsdifferenz. Leider sagen sie gerade über das Drehungsvermögen des Osazons nichts. Mit Rücksicht darauf, daß sie zum Diphenylhydrazon nicht gelangen konnten, weisen sie darauf hin, daß es sich bei der Harnpentose um $d, l$-Ribose handeln könnte. Dieser Schluß schien ihnen umso verlockender als ja durch Levene und Jakobs die Natur der Organopentose,

1 Pflüger's Archiv, 110, 625 (1905).

2 Zeitschr. f. klin. Medisin, 59, 246 (1906).

$\Im$ Journ. of biolog. Chemistry, XI, 213 (1912). 
zumindest der der Inosinsäure, als $d$-Ribose ziemlich sicher bewiesen worden ist.

Kurz vor Elliot und Raper hat sich auch noch der Schwede Klercker ${ }^{1}$ mit dem Studium zweier Pentosuriefälle in der eingehendsten Weise befaßt. Er konnte in einem Fall ein Osazon, das in der von $\mathrm{Neuberg}$ angegebenen Konzentration $\left(0.2 \mathrm{~g}\right.$ in $10 \mathrm{~cm}^{3}$ Pyridin-Alkohol) bei Auerlicht etwa $0.6^{\circ}$ nach rechts drehte, isolieren; für $l$-Arabinosazon aus $l$-Arabinose verschiedener Provenienz ermittelte er den annähernd gleichen Wert. Das Osazon aus dem anderen Pentoseharn drehte gleichfalls deutlich rechts, jedoch nur halb so stark; zu bemerken wäre, daß in diesem Fall neben der Pentose sehr reichliche Mengen (bis zu $1.1 \%$ ) Glukose ${ }^{2}$ ausgeschieden wurden. Eine Isolierung der Pentose gelang nicht, insbesondere auch nicht nach dem Neuberg'schen Diphenylhydrazinverfahren. Klercker kommt, wohl befangen durch den Neuberg'schen Befund, zu dem Schluß, daß es sich in dem einen seiner Fälle um reine $l$-Arabinose handle, im anderen um ein Gemisch der $d$ - und l-Form dieses Zuckers, in welchem letztere überwiegt.

Die beiden Pentosuriefälle, mit denen wir uns beschäftigt haben, stammen aus der v. Noorden'schen Klinik. Herr Dr. Otto Porges, Privatdozent und Assistent an dieser Klinik, hat die Freundlichkeit gehabt, uns mehrere Liter gesammelten Urins zur näheren Untersuchung zu überlassen, wofür wir ihm auch an dieser Stelle unseren herzlichsten Dank aussprechen. Über Fall I berichtete er uns folgendes:

"S. R. 28jähriger Mann, trat zum erstenmal 1912 als Diabetiker auf die Klinik ein. Somatisch nichts Pathologisches, hochgradige Neurasthenie. Bei Beobachtung des angeblichen Diabetes zeigte sich, daß diätetische Maßnahmen ohne Einfluß auf die Zuckerausscheidung sind. Diese bleibt vielmehr bei kohlehydratfreier und gemischter Kost annähernd gleich und beträgt zirka $0.5 \%$ (Reduktion nach Citron, gerechnet als Glukose). Daher wurde auf Pentosurie geprüft. Tatsächlich gab

1 L. c.

2 Wiewohl Klercker das Osazon bloß aus heißem Wasser umkrystallisiert hat, ist die Beimengung von Glukosazon gewiß müglich, da ja die beiden Osazone sicherlich gegenseitig ihre Löslichkeit beeinflussen. 
der Harn die bekannten Pentosefarbenreaktionen, positive Fehling'sche, schwache Nylander'sche Reaktion und zeigte keine Drehung. Das Osazon zeigte nach mehrmaligem Umkrystallisieren aus verdünntem Aceton den Schmelzpunkt $158^{\circ}$. $0.2 \mathrm{~g}$ in dem üblichen Pyridin-Alkoholgemisch gelöst, drehten $+0.75^{\circ}$ (Natriumlicht). Weder durch Traubenzucker, noch durch gehäufte Fleischdiät, noch durch Hefenucleinsäure wurde eine wahrnehmbare, alimentäre Beeinflussung der Zuckerausscheidung erzielt. Einmal wurden auch $40 \mathrm{~g}$ Galaktose gegeben. Die Zuckermenge stieg von $0.56 \%$ des Vortages in dem Urin aus den ersten 2 Stunden nach Eingabe auf $1.82 \%$, jedoch zeigte der Harn im Polarimeter $1.5 \%$ Rechtsdrehung, es ist also hauptsächlich unverbrauchte Galaktose vorhanden. Die weiteren Harne waren dann wieder inaktiv und die Zuckermenge unerheblich größer als sonst $(0.6$ bis $0.9 \%)$. Bei einer ein Jahr später erfolgten Untersuchung dieses Patienten war der Harnbefund unverändert. «

Der uns übergebene Harn reagierte schwach sauer, zeigte das spezifische Gewicht 1.025, war von goldgelber Farbe und lieferte die bekannten Pentosereaktionen in normaler Weise. Die quantitative Bestimmung des Zuckers durch Reduktion nach Allihn ergab in $25 \mathrm{~cm}^{3} 0.1658 \mathrm{~g}$ Kupfer entsprechend einem Gehalt von $0.34 \%$ (als Glukose). Außerdem wurde die Pentose auch nach Tollēns durch Destillation mit Salzsäure und Wägen des Furfurols als Phloroglucid bestimmt. Aus $100 \mathrm{~cm}^{3}$ Harn erhielten wir $0 \cdot 1238 \mathrm{~g}$ Phloroglucid, entsprechend $0 \cdot 135 \%$ Arabinose. Daß die Reduktion annähernd doppelt so große Werte liefert als die Furfurolbestimmung, hat auch Klerker bei seinen beiden Fällen beobachtet und es ist daher die Annahme von Neuberg, ${ }^{1}$ daß die bei Harnpentosen eintretende schußartige Reduktion, auf die Bindung der Pentose an Harnstoff zurückzuführen sei, auch in unserem Falle gewiß unbegründet. Das Osazon ließ sich aus dem Harne direkt bereiten. Es fiel in der Hauptsache erst beim Abkühlen aus, auch genügte einstündiges Erhitzen nicht, denn man erhält bei Wiederholung des Erhitzens noch immer neuen Niederschlag.

1 Ergebnisse der Physiologie, Bd. III, Abt. I, 418 (1904). 
Bei einer quantitativ erfolgten Bereitung erhielten wir eine Osazonmenge entsprechend $0.15 \%$ Zucker. Derartige erhebliche Differenzen der Zuckerbestimmung aus Osazonmenge und Reduktionsvermögen hat auch Salkowski ${ }^{1}$ beobachtet. Da Luzzatto sagt, daß sein Osazon beim Umkrystallisieren aus verdünntem Alkohol stets wechselnde analytische Werte gab, ohne daß sich der Schmelzpunkt wesentlich änderte, einen Übelstand, den er erst durch Umkrystallisieren aus wässerigem Pyridin beheben konnte, haben wir verschiedene Fraktionen der Analyse unterworfen. Zunächst wurde das Osazon zweimal aus 20 prozentigem Aceton umkrystallisiert; es zeigte den Schmelzpunkt 162 bis $163^{\circ}$ (Erhitzungsdauer 5 Minuten).

I. 9.57 $\mathrm{mg}$ Substanz, im Toluolbad getrocknet, gaben nach Pre g1 $1.411 \mathrm{~cm}^{3}$ Stickstoff $\left(22^{\circ}, 754 \mathrm{~mm}\right)$.

Ii. 11.06 $\mathrm{mg}$ Substanz, im Toluolbad getrocknet, gaben nach Preg1 1. $622 \mathrm{~cm}^{3}$ Stickstoff $\left(22^{\circ}, 754 \cdot 5 \mathrm{~mm}\right)$.

In 100 Teilen:

\begin{tabular}{|c|c|c|c|}
\hline & \multicolumn{2}{|c|}{ Gefunden } & \multirow{2}{*}{$\begin{array}{l}\text { Berechnet fü } \\
\mathrm{C}_{17} \mathrm{H}_{20} \mathrm{O}_{3} \mathrm{~N}_{4}\end{array}$} \\
\hline & I. & II. & \\
\hline 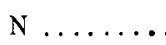 & $16 \cdot 93$ & $16 \cdot 85$ & $17 \cdot 07$ \\
\hline
\end{tabular}

Dieses Produkt wurde nochmals aus 20 prozentigem Aceton umkrystallisiert; der Schmelzpunkt blieb unverändert. Die analytischen Resultate dieser Fraktion waren die Folgenden:

I. 4.68 $\mathrm{mg}$ Substanz, im Toluolbad getrocknet, gaben nach Preg1 10.64 $\mathrm{mg}$ Koblensäure und $2 \cdot 77 \mathrm{mg}$ Wasser.

II. 4.96 $\mathrm{mg}$ Substanz, im Toluolbad getrocknet, gahen nach PregI $0 \cdot 7536 \mathrm{~cm}^{3}$ Stickstoff $\left(22^{\circ}, 754 \mathrm{~mm}\right)$.

In 100 Teilen:

\begin{tabular}{|c|c|c|c|}
\hline & \multicolumn{2}{|c|}{ Gefunden } & \multirow{2}{*}{$\begin{array}{l}\text { Berechnet für } \\
\mathrm{C}_{17} \mathrm{H}_{20} \mathrm{O}_{3} \mathrm{~N}_{4}\end{array}$} \\
\hline & I. & II. & \\
\hline$C \ldots \ldots \ldots$ & $62 \cdot 01$ & - & $62 \cdot 20$ \\
\hline $\mathrm{H} \ldots$ & $6 \cdot 61$ & - & $6 \cdot 10$ \\
\hline $\mathbf{N} \ldots \ldots \ldots$ & - & $17 \cdot 40$ & $17 \cdot 07$ \\
\hline
\end{tabular}

1 I., c. 
Durch das Umkrystalisieren haben sich also weder der Schmelzpunkt noch die analytischen Werte geändert. Bei längerem Liegen (nach etwa 2 Monaten) sind die Osazonpräparate etwas dunkler geworden und der Schmelzpunkt um zirka $2^{\circ}$ heruntergegangen.

Von dem obigen Präparate wurde auch eine Drehungsbestimmung gemacht; diese, wie auch alle anderen polarimetrischen Bestimmungen wurden in einem modernen Halbschattenapparat der Firma Schmidt und Hänsch mit dreiteiligem Gesichtsfeld, der eine Ablesung von $0.01^{\circ}$ gestattet, ausgeführt.

$0.0952 \mathrm{~g}$ Osazon in $10 \mathrm{~cm}^{3}$ Pyridin-Alkohol, $5 \frac{1}{2}$ Stunden nach der Auflösung polarisiert, drehen bei Natriumlicht +0.34 $\pm 0.02^{\circ}$. Das gibt für die von Neuberg angegebene Konzentration

$$
\alpha_{D}=+0 \cdot 71 \pm 0 \cdot 04^{\circ} .1
$$

Der Harn selbst zeigte im $220 \mathrm{~mm}$ Rohr keine Drehung.

Wir hatten also gleich Luzzatto und K1ercker den Fall einer Pentosurie mit inaktivem Harn und rechts drehendem Osazon. Allerdings ist die Größe der von uns gefundenen Drehung bedeutend kleiner als dem reinen $l$-Arabinosazon nach $\mathrm{N}$ e u b e r $\mathrm{g}^{2}$ zukommt, das wäre $117^{\circ}$. Aber die Angaben hierüber differieren recht wesentlich. Leven $e^{3}$ fand für $l$-Arabinosazon und $l$-Xylosazon die Neuberg'schen Werte, für das Pentosazon aus Hefenucleinsäure hingegen $-0 \cdot 84^{\circ},{ }^{3}$ für das Pentosazon aus Pankreas, die beide nach seinen Arbeiten mit $d$-Arabinosazon identisch sein sollen, $-0 \cdot 94^{\circ}{ }^{4}$ Haiser und Wenze1 $1^{5}$ finden für das Osazon des Zuckers aus Inosinsäure unmittelbar nach der Auflösung $-1 \cdot 10^{\circ}, 18$ Stunden später nur mehr $-0 \cdot 82^{\circ} .{ }^{6}$ Klercker ${ }^{7}$ beobachtete bei Auerlicht für verschiedene Präparate von $l$-Arabinosazon $+0.69^{\circ},+0.62^{\circ}$ und $+0.68^{\circ}$, während

1 Das Pyridin-Alkoholgemisch war selbstverständlich vorher als inaktiv befunden worden.

2 B. 32, 3384 (1899).

3 Biochem. Zeitschr, 17, 127 (1909).

4 Levene und Jakobs, B. 43, 3150 (1910).

5 M. 31, 360 (1910).

6 Die von den Autoren in verschiedenen Konzentrationen ermittelten Drehungen sind auf $0.2 \mathrm{~g}$ in $10 \mathrm{~cm}^{3}$ Pyridinalkohol im $1 \mathrm{dm}$-Rohr umgerechnet.

7 Biochem. Zeitschr. 47, 340 (1912). 
endlich wir selbst für ein Osazon aus $l$-Arabinose Merck (Schmp. 152 bis $155^{\circ}$ ) $+0 \cdot 85^{\circ}$ ( 6 Stunden nach der Auflösung) ablesen konnten. Diese relativ großen Unterschiede bei Beobachtung der gleichen Erscheinung sind, wie uns scheint, durch zwei Umstände zu erklären: Zunächst durch die Multirotation, die durch die Angaben Haiser's und Wenzel's bewiesen ist, die übrigens auch von Levene und Jakob's ${ }^{1}$ beim $p$-Bromphenylosazon der Arabinose konstatiert wurde, entgegen der Neuberg'schen Angabe, daß im Pyridin-Alkoholgemisch Multirotation nicht auftrete. Dann dürfte aber auch die genaue Zusammensetzung des Pyridin-Alkoholgemisches eine gewisse Rolle spielen, worauf ja Neuberg selbst, insbesondere bei der Drehungsbestimmung der Verbindung aus Glukuronsäure und $p$-Bromphenylhydrazin, hinweist.

Wir haben uns mit diesem für uns sekundären Moment nicht eingehender beschäftigt, weil wir ebenso wie Klercker in seiner bereits zitierten Arbeit, zu der Überzeugung gekommen sind, daß durch die Bestimmung der Größe des Drehungsvermögens allein eine Unterscheidung zwischen Xylosazon und Arabinosazon nicht getroffen werden kann. Denn während $\mathrm{Neuberg}{ }^{2}$ in Übereinstimmung mit einigen anderen Autoren für ersteres $-0.25^{\circ}$ angibt, fand Klercker ${ }^{3}-0.67$ und $-0 \cdot 70^{\circ}$, wir selbst für ein Präparat aus $l$-Xylose (Kahlbaum, Schmp. 141 bis $\left.146^{\circ}\right)-0.66 \pm 0.06^{\circ}$. Es mußte daher ein anderes sicheres Mittel gesucht werden, das entscheiden konnte, welchem Pentosentypus vorliegendes Osazon entstamme.

Wir wollen wieder zu der Harnpentose zurückkehren. Wir haben früher hervorgehoben, daß in mehreren Fällen aus inaktiven Pentosenharnen ein aktives Osazon von dem annähernden Drehungsvermögen des $l$-Arabinosazons gewonnen werden konnte. Daraus wurde von mehreren Seiten der Schluß gezogen, daß es sich hier um $l$-Arabinose handle. Dieser Schluß war zweifellos von dem Resultate Neuberg's beeinflußt. Bei reiflicher Überlegung mußten wir uns aber sagen, daß die Fälle

\footnotetext{
1 B. 42,3249 (1909).

2 B. 32, 3384 (1899).

3 Biochem. Zeitschr. 47, 340 (1912).
} 
von Pentosurie mit aktivem Osazon mit dem Neuberg'schen Falle gar nichts zu tun haben können, dieser vielmehr eine ausgesprochene Sonderstellung einnehmen muß. Um $l$-Arabinose kann es sich in diesen Fällen aus zwei Gründen nicht handeln. Der erste, vielleicht minder ins Gewicht fallende ist, ${ }^{1}$ daß es niemand gelungen ist, aus den Harnen ein Diphenylhydrazon $\mathrm{zu}$ isolieren, wiewohl Neuberg und Wohlgemuth ${ }^{2}$ ein Verfahren zur quantitativen Bestimmung der Arabinose im Harn mit Diphenylhydrazin ausgearbeitet und genau ausgeprüft haben. Wir selbst konnten ebensowenig, nach Neuberg's Vorschrift arbeitend, zu einem Diphenylhydrazon gelangen. In neuerer Zeit ist aber ein noch viel empfindlicheres Reagens auf Arabinose von J. v. Braun ${ }^{3}$ angegeben worden, das Diphenylmethandimethyldihydrazin. Nach Angabe dieses Autors läßt sich bereits aus $0.2 \%$ Arabinose enthaltendem Harn quantitativ das entsprechende Hydrazon gewinnen und wir haben uns selbst davon überzeugt, daß man aus normalem Harn, dem $0.3 \%$ Arabinose zugesetzt waren, in 3 bis 4 Tagen glatt und annähernd quantitativ das Hydrazon mit den v. Braun angegebenen Eigenschaften erhält. Hingegen konnten wir aus unserem Pentoseharn, der an und für sich konzentrierter war, und den wir zu diesem Zweck noch auf das halbe Volum einengten, mittels dieses ausgezeichneten Reagens nur sehr geringe Quantitäten eines Niederschlages erhalten, der von dem Braunschen Körper wesentlich verschieden ist und aus dem sich durch die übliche Benzaldehydspaltung ${ }^{4}$ kein Produkt von reduzierenden Eigenschaften gewinnen ließ. Einen recht ähnlichen Niederschlag, allerdings in etwas geringerer Menge, haben wir auch aus zuckerfreiem Harn mit Diphenylmethandimethyldihydrazin bekommen; derselbe stellt also wohl in der Hauptsache Oxydations- oder andere Zersetzungsprodukte des Hydrazins vor.

1 Dieser Umstand hat, wie erwähnt, auch schon Elliot und Raper bewogen, an der Arabinosenatur der Harnpentose zu zweifeln.

2 H. 35,38 (1902).

3 B. $43,1502(1910)$.

4 Es resultierte bloß eine Lösung, die schwache Pentosephloroglucinreaktion gab. 
Ein zweiter absolut beweisender Umstand schließt jedoch in den inaktiven Harnen mit aktivem Osazon das Vorhandensein der l-Arabinose unbedingt aus: die Inaktivität des Harnes. Gerade die Arabinose hat ein sehr hohes spezifisches Drehungsvermögen; es beträgt etwa $104^{\circ}$, ist also annähernd doppelt so groß als das des Traubenzuckers. Und da man im Harn $0.1 \%$ Glukose polarimetrisch noch mit Sicherheit bestimmen kann, könnte doch die Drehung von $0.3 \%$ Arabinose, wie sie z. B. Klercker durchschnittlich in seinen Fällen gefunden hat und wie sie auch bei uns vorliegt, und auch viel geringere Arabinosekonzentrationen, unmöglich zu übersehen sein. Daß andere Harnbestandteile diese Drehung soweit zurückdrängen können, ist nicht nur erfahrungsgemäß unwahrscheinlich, es ist auch speziell für Arabinose durch Neuberg und Wohlgemuth widerlegt worden. Diese Autoren sagen selbst in einer Arbeit, die sich mit der Ausnutzung von $d$ - und l-Arabinose im Kaninchenkörper befaßt: "Man kann die quantitative Bestimmung der racemischen Arabinose ${ }^{1}$ unbedenklich auf die polarimetrische Ermittlung gründen, umsomehr als die spezifische Drehung der Arabinose sehr groß ist, nämlich $[\alpha]_{D}= \pm 104- \pm 106^{\circ}{ }{ }^{2}{ }^{2}$

Wir waren daher von vornherein der Ansicht, daß es sich in unseren, wie in den ähnlichen Pentosuriefällen anderer Autoren wahrscheinlich um $l$-Ribose handeln würde, deren Drehungsvermögen ja nur rund sechsmal so klein als das der Arabinose, dreimal so klein als das der Glukose ist. Zur Sicherstellung dieses Schlusses wäre es nur noch notwendig gewesen zu zeigen, daß das Harnpentosazon wirklich $l$-Arabinosazon ist.

Wir sind damit zu der schon einmal aufgeworfenen Frage zurückgekehrt, wie man Xylosazon und Arabinosazon von-

1 Die Gesamtmenge an Arabinose wurde dabei nach dem mehriach erwähnten Diphenylhydrazinverfahren bestimmt.

$2 \mathrm{DaB}$ etwa die Arabinose in gebundenem Zustand vorliegt und der Komplex nur ein kleines Drehungsvermögen zeigt, ist in unserem Fall ausgeschlossen, weil, wie wir schon oben sagten, die durch Redultion ermittelten Zuckerwerte stets erheblich größer waren als die nach der Furfurolmethode erhaltenen. 
einander unterscheiden kann. Die Drehung allein ist nach unseren früheren Ausführungen nicht charakteristisch. Wir hätten eventuell das $p$-Bromphenylosazon heranziehen können, da nach neueren Angaben ${ }^{1}$ dieses Derivat der Arabinose um etwa $20^{\circ}$ tiefer schmilzt als das der Xylose, allein die Bromphenylosazone sind nach übereinstimmenden Angaben, viel schwerer rein und krystallisiert zu erhalten, als die gewöhnlichen Osazone.

Es schien uns jedoch möglich, vielleicht durch ein viel einfacheres Mittel $\mathrm{zu}$ entscheiden, ob ein rechtsdrehendes Pentosazon dem Xylose- oder Arabinosetypus entstammt, nämlich die Methode des Mischschmelzpunktes. Nach Arbeiten von E. Fischer" schmilzt das $d, l$-Xylosazon bei 210 bis $215^{\circ}$. Mischt man also das fragliche rechtsdrehende Osazon mit (linksdrehendem) 7.Xylosazon und es tritt eine wesentliche Erhöhung des Schmelzpunktes ein, dann muß $d$-Xylosazon vorliegen. Zeigt der Mischschmelzpunkt aber keine wesentliche Veränderung oder eine Depression, dann könnte $l$-Arabinosazon vorhanden sein. Wir haben diese gewiß sehr einfache Probe ausgeführt und zu unserer Überraschung gab ein aus annähernd gleichen Teilen $l$-Xylosazons und unseres Osazons hergestelltes Gemisch einen Schmelzpunkt von etwa $200^{\circ}$. Es mußte also unser Osazon aus Harn $d$ Xylosazon sein.

Die bei beiden Osazonen, die also optische Antipoden vorstellen, abgelesene Drehung ist tatsächlich annähernd gleich. Wir fanden für $l$-Xylosazon $-0.66 \pm 0.06^{\circ}$, für unser Harnpentosazon $+0.71 \pm 0.04^{\circ}$. Zum endgültigen Beweis haben wir abgewogene gleiche Mengen beider Osazone vereint aus 20 prozentigem Aceton umkrystallisiert. Es zeigte sich, daß das Gemisch erheblich schwerer löslich ist, als die beiden Komponenten.

Von dem aus der Lösung auskrystallisierten Osazon einerseits, $l$-Xylosazon und unserem Harnpentosazon andrerseits, wurden nebeneinander die Schmelzpunkte bestimmt.

1 Rewald, B. 42, 3135 (1909); Levene und Jakobs, B. 42, 3249 (1909); Steudel und Brigl, H. 68, 49 (1910).

2 B. 27, 2488 (1894); Fischer und Ruff, B. 33, 2145 (1900). 
l-Xylosazon

162 bis $163^{\circ}$
Harnpentosazon

160 bis $161^{\circ}$ gemeinsam umkrystallisiert:

202 bis $203^{\circ}$

(10 Minuten Erhitzungsdauer.)

Bei nur 5 Minuten langem Erhitzen war der Schmelzpunkt des »Mischosazons « 208 bis $210^{\circ}$, also in guter Übereinstimmung mit der von E. Fischer angegebenen Zahl 210 bis $215^{\circ}$. Wir haben auch konstatiert, daß $0 \cdot 1266 \mathrm{~g}$ dieses Mischosazons in $10 \mathrm{~cm}^{3}$ Pyridin-Alkohol (die Mischung enthielt etwas mehr Pyridin, da im üblichen Gemisch nicht Lösung eintrat) im $1 \mathrm{dm}$ Rohr keine Drehung zeigte.

Wie bereits erwähnt, wurde uns auch noch ein zweiter Pentoseharn zur Untersuchung überlassen. Über diesen berichtet uns Herr Porges folgendes:

Fall II. " 52 jährige Frau, außer Fettleibigkeit nichts Pathologisches. Da geringe Zuckermengen bei ihr beobachtet worden waren, wurde sie auf die Klinik aufgenommen. Im Harne wurden 0.1 bis $0.2 \%$ Zucker gefunden (Reduktion, gerechnet als Glukose). Zuckerausscheidung auch hier alimentär nicht zu beeinflussen. Daher wurde auf Pentose geprüft. Farbenreaktionen positiv. Osazon $156^{\circ}$ (vielleicht nicht ganz rein). Harn inaktiv, Drehung des Osazons hier nicht untersucht. Diese Patientin bekam an einem Tag $50 \mathrm{~g}$ Hefenucleinsäure, ohne $\mathrm{da} \beta$ eine Vermehrung der Zuckerausscheidung eintrat.*

Wir haben uns bei diesem zuckerarmen Harn darauf beschränkt, das Osazon herzustellen und $z u$ untersuchen. Das Osazon, welches aus dem zuvor im Kohlensäurestrom in vacuo konzentrierten Harn gewonnen und dann dreimal aus 20 prozentigem Aceton umkrystallisiert worden war, schmolz bei 161 bis $162^{\circ}$ und erwies sich auch durch die Stickstoff bestimmung als Pentosazon.

8. $11 \mathrm{mg}$ Substanz gaben nach Pregl $1 \cdot 23 \mathrm{~cm}^{3}$ Stickstoff $\left(20^{\circ}, 743 \mathrm{~mm}\right)$.

In 100 Teilen:

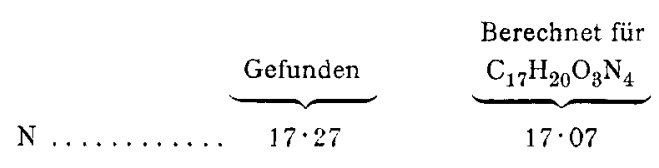


Von der Inaktivität des Harnes haben wir uns überzeugt, die Drehung des Osazons konnten wir nicht untersuchen, da eine Lösung von $0.075 \mathrm{~g}$ in $15 \mathrm{~cm}^{3}$ Pyridin-Alkohol bereits als zu dunkel für die Polarisation ungeeignet war. Es ist das aber auch völlig überflüssig. Denn auch dieses Osazon gibt, zu annähernd gleichen Teilen mit $l$-Xylosazon gemischt, einen Mischschmelzpunkt von zirka $195^{\circ}$.

Dadurch ist erwiesen, daB in beiden Fällen das Osazon aus Pentoseharn $d$-Xylosazon ist und es kann dementsprechend die zugrundeliegende Pentose nur mehr die drei folgenden Konfigurationen haben:

\begin{tabular}{ccc}
$\mathrm{C}_{\mathrm{H}}^{\mathrm{O}}$ & \multicolumn{1}{c}{$\mathrm{O}$} & $\mathrm{CH}_{2} \mathrm{OH}$ \\
$\mathrm{HOCH}$ & $\mathrm{HCOH}$ & $\mathrm{CO}$ \\
$\mathrm{HCOH}$ & $\mathrm{HCOH}$ & $\mathrm{HCOH}$ \\
$\mathrm{HOCH}$ & $\mathrm{HOCH}$ & $\mathrm{HOCH}$ \\
$\mathrm{CH}_{2} \mathrm{OH}$ & $\mathrm{CH}_{2} \mathrm{OH}$ & $\mathrm{CH}_{2} \mathrm{OH}$ \\
$d$-Xylose & $l$-Lyxose & $d$-Xyloketose
\end{tabular}

Es schien anfänglich leicht, nunmehr auch die Natur des Zuckers selbst $\mathrm{zu}$ ermitteln. Die Xyloketose war von vornherein der Furfurolausbeute wegen unwahrscheinlich, es blieb also nur die Feststellung übrig, ob es sich um $d$-Xylose oder die noch unbekannte $l$-Lyxose handle. Da nun die $d$-Xylose links, die $l$-Lyxose aber rechts dreht, so mußte es genügen, die Drehungsrichtung des konzentrierten Harnes zu beobachten. Wir haben das versucht, indem wir etwa $2 l$ Harn mit einem Überschuß von Bleizuckerlösung fällten und dann im Vakuum im Kohlensäurestrom bei einer $36^{\circ}$ nicht übersteigenden Temperatur konzentrierten. Die sehr dunkle Lösung (zirka $100 \mathrm{~cm}^{3}$ ) wurde dann mit Schwefelwasserstoff entbleit, aber dieses Filtrat war für die polarimetrische Untersuchung viel zu dunkel. Diesen Übelstand zu beheben, ist uns in keiner Weise gelungen. Weder Behandeln mit Tierkohle, noch mit gereinigter Kieselgur und nachherige Filtration durch ein Pukallfilter führten zum Ziele. Etwas glücklicher waren wir bei einer zweiten Portion von $2 l$, die gleichfalls mit Bleiacetat gefällt, dann aber nur auf ein Viertel konzentriert worden war. Hier konnten wir im $1 \mathrm{dm}$-Rohr eine Drehung von $-0.08^{\circ}$ ablesen, so daß das 
Vorhandensein von $d$-Xylose recht wahrscheinlich ist. Immerhin möchten wir diese Behauptung nur mit aller Reserve aufstellen. Denn die Bestimmung des Reduktionsvermögens hat mit der durch die obige Drehungsbestimmung ermittelten Zuckerkonzentration keine Konkordanz ergeben. Aus der Drehung berechnet sich für $[a]_{D}=-18.8^{\circ}$ (Xylose) eine Zuckermenge von $0.43 \%$, während die Reduktion nach Allihn $0 \cdot 65 \%$ ergab.

Da wir immerhin allen Grund hatten anzunehmen, daß es sich um $d$-Xylose handle, haben wir natürlich versucht, das von Bertrand ${ }^{1}$ beschriebene Bromcadmiumdoppelsalz der Xylonsäure aus unserem Pentoseharn zu gewinnen. $\mathrm{Zu}$ diesem Ende wurde der mit Bleiacetat gefällte Harn sehr stark im Vakuum konzentriert und dann zweimal in heißen Alkohol gegossen. Der Rückstand der alkoholischen Lösung wurde dann mit Brom oxydiert, jedoch gelang es uns nicht, das charakteristische Doppelsalz zu erhalten.

Sicher ist also, daß der Zucker in beiden Pentösuriefällen dem $d$-Xylosetypus angehört, nicht unwahrscheinlich, daß er d-Xylose ist. Da wir hoffentlich noch eine größere Menge Harn ron diesem Patienten (Fall I) erhalten werden, wird sich diese letztere Annahme wohl noch exakt durch das Experiment beweisen lassen.

1 Bull. 5, $556(1891)$. 